\title{
Développements récents sur les détecteurs cryogéniques, applications dans la recherche et l'industrie
}

\author{
E. LEBLANC ${ }^{1}$
}

(Manuscrit reçu le 12 février 2004, accepté le 8 juillet 2004)

RÉSUMÉ Les performances remarquables des détecteurs fonctionnant à très basse température ont franchi les limites inhérentes aux détecteurs conventionnels à semiconducteur ou à scintillateur. Ceci s'explique par les principes physiques de détection exploités par les différentes technologies développées aujourd'hui. L'élégance de ces technologies est de mettre en jeu la création de quanta d'énergie inférieure de trois à quatre ordres de grandeur par rapport à celle des détecteurs classiques. Les très faibles fluctuations statistiques du nombre de quanta créés pour une interaction donnent ainsi accès à des seuils de détection et des résolutions en énergie meilleurs de un à deux ordres de grandeur. De plus le rendement de détection n'est pas limité à basse énergie comme dans le cas des semi-conducteurs en germanium ou en silicium. Les détecteurs cryogéniques sont aujourd'hui développés et utilisés dans des applications multiples (caméras pour télescopes, spectromètres imageurs, analyse de matériaux par fluorescence $X$, mesure d'activité, ...), qui recouvrent la détection des rayonnements sub-millimétriques, infrarouges, UV, X, gamma, bêta et des particules alpha. L'apparition très récente sur le marché de réfrigérateurs sans liquide cryogénique et très simples d'utilisation ouvre de nouvelles perspectives d'applications industrielles de ces détecteurs.

ABSTRACT Cryogenics detectors: recent developments and applications in research and industry.

The performance of low temperature detectors has far exceeded the inherent limits of conventional semiconductor and scintillation detectors. This stems from the detection physics used by the different technologies being developed nowadays. The neat idea behind these technologies is to split the energy of an incident photon or particle over a very large number of quanta, larger by several orders of magnitude than in classical detectors. Hence low statistical fluctuations on the energy determination for an individual interaction lead to energy detection thresholds and energy resolutions better by one or two orders of magnitude. An additional asset is that detection efficiency is not limited at low energies as it is the case for germanium or silicon detectors. Low temperature detectors are being developed and used in numerous applications (imaging arrays for astrophysics, dark matter and neutrino physics, atomic, nuclear and particle physics, material science, biology and medical science, ...) covering detection of different nature of particles over a very large energy range (sub-millimetre, infra-red, UV, X-rays, gamma photons, but also beta, alpha particles, and bio-molecules). Introduction of automated cryogen-free refrigerators on the market opens new prospects of industrial applications for these detectors of hitherto unequalled performance.

\footnotetext{
1 Bureau national de métrologie, Laboratoire national Henri Becquerel, CEA-Saclay, 91191 Gif-sur-Yvette, France.
} 


\section{Introduction}

Un rayonnement ionisant (photon $\mathrm{X}$ ou gamma, électron, particule alpha,...) interagit avec la matière principalement en cédant son énergie dans un temps relativement court (picoseconde) aux électrons du milieu. Suivant la nature du matériau dans lequel a lieu l'interaction, une partie de cette énergie se retrouve sous forme d'atomes ionisés dans un gaz par exemple, ou de paires électron-trou dans un semi-conducteur, ou de photons lumineux dans le cas d'un scintillateur. Une partie de l'énergie peut également être piégée dans des défauts de structure ou par des impuretés. Dans la plupart des cas, la plus grande partie de l'énergie disponible est convertie en chaleur.

Chaque classe de détecteur de rayonnements ionisants exploite de façon privilégiée un des phénomènes physiques précédents. Parmi les détecteurs classiques par exemple, les compteurs proportionnels sont fondés sur l'ionisation, les semi-conducteurs sur la création de paires électron-trou, les scintillateurs sur la production de photons lumineux. Dans les cas cités, seulement une partie de l'énergie du rayonnement incident est convertie en énergie détectable, alors que la plupart est dissipée en chaleur.

Quel que soit le principe physique de détection, l'énergie détectable est quantifiée. Plus le nombre de quanta ou de porteurs d'énergie est important, plus les fluctuations statistiques sur la mesure de cette énergie sont faibles. Par conséquent les détecteurs qui favorisent la distribution de l'énergie sur un très grand nombre de porteurs d'énergie, donnent accès à des seuils de détection très bas et à une meilleure résolution en énergie. Par exemple, dans un scintillateur NaI, l'énergie d'un rayonnement incident de $6 \mathrm{keV}$ sera répartie sur seulement quelques dizaines de photons lumineux. Alors que la même énergie dans un semiconducteur en germanium ou en silicium sera distribuée sur environ $10^{3}$ paires électron-trou. Ceci explique pourquoi les détecteurs en germanium ou en silicium ont apporté dans les années 1970 une révolution des performances par rapport aux détecteurs $\mathrm{NaI}$.

Pour introduire une rupture technologique dans la mesure des rayonnements ionisants et franchir de plusieurs ordres de grandeur les limites imposées par la physique des détecteurs conventionnels, la question qui se pose est la suivante : existe-t-il une autre forme d'énergie détectable que celles mises en jeu dans les détecteurs classiques et qui soit distribuée sur un nombre encore plus grand de porteurs d'énergie ? Une réponse positive à cette question peut être trouvée à très basse température. Suivant la nature du matériau, supra-conducteur, diélectrique ou métal, l'énergie peut être distribuée sur un très grand nombre de porteurs d'énergie. Les détecteurs mettant en jeu une mesure de ces quanta à des 


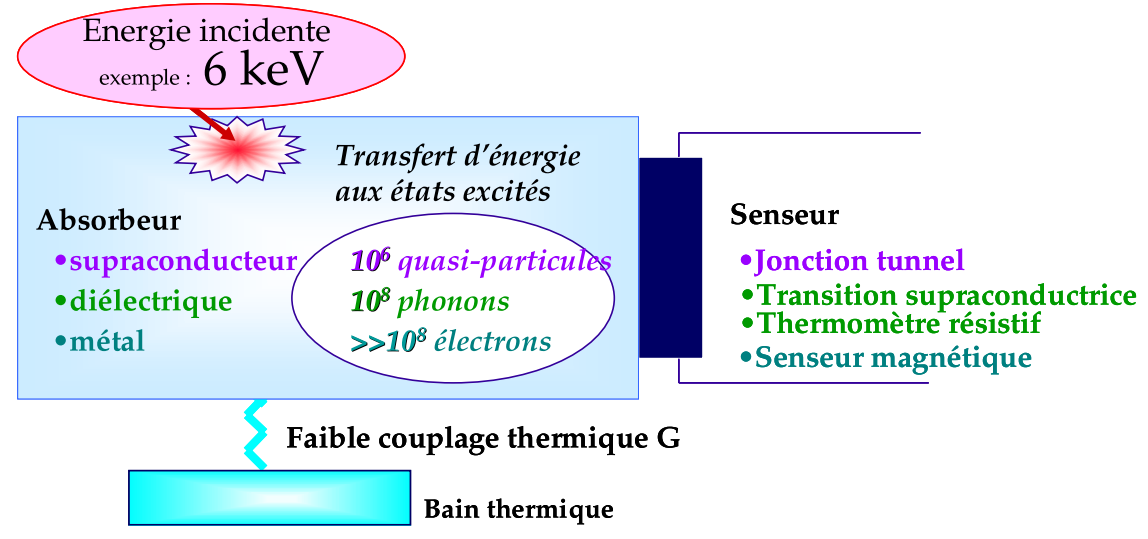

Figure 1 - Détecteurs cryogéniques développés aujourd'hui : trois principes de détection sont exploités. Low temperature detectors: detection can be based on excitation of quasi-particles, phonons, or electrons.

températures inférieures à quelques centaines de millikelvin sont communément désignés par les termes de «détecteurs cryogéniques ». Derrière cette expression se rassemble une diversité de technologies exploitant des principes physiques très différents.

\section{Détecteurs cryogéniques : différentes technologies fondées sur des principes physiques différents}

L'idée à l'origine des détecteurs cryogéniques est, d'une part de convertir la plus grande partie de l'énergie d'un rayonnement incident sous forme de chaleur et, d'autre part de distribuer cette énergie sur un très grand nombre de porteurs d'énergie pour mesurer cette quantité avec de faibles fluctuations statistiques.

Il existe des détecteurs cryogéniques très différents, néanmoins, on retrouve trois éléments communs à tous ces détecteurs représentés sur la figure 1 :

- un absorbeur ou une cible dans laquelle le rayonnement incident interagit et cède son énergie en excitant des porteurs d'énergie,

- un senseur qui va permettre de mesurer le nombre de porteurs d'énergie, ce nombre étant proportionnel à l'énergie cédée par le rayonnement incident,

- un faible couplage thermique avec un bain thermique qui après chaque interaction, réinitialise le détecteur (permet sa désexcitation). 

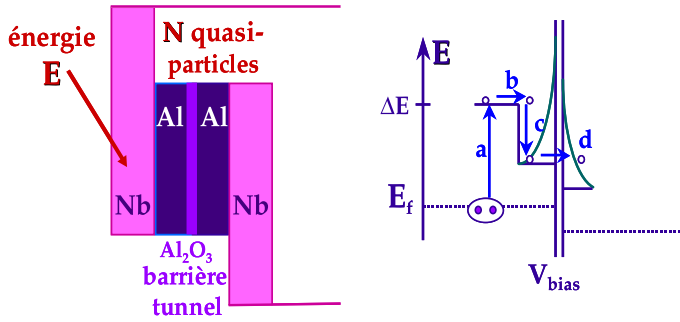

a : brisure des paires de Cooper, $b$ : diffusion des quasi-particules c : piégeage des quasi-particules d : passage par effet tunnel

Figure 2 - Jonction tunnel supraconductrice.

Superconducting tunnel junction.

Quels sont donc ces porteurs d'énergie en très grand nombre que l'on peut mesurer directement? Ils diffèrent suivant la nature du matériau. Trois principes physiques sont aujourd'hui exploités ; ils classent les détecteurs cryogéniques parmi trois technologies principales.

\subsection{Transfert d'énergie aux quasi-particules}

Dans un supraconducteur, à très basse température (inférieure à une centaine de millikelvin pour la plupart des matériaux supra-conducteurs), les électrons de conduction sont couplés en paires de Cooper. L'énergie d'un rayonnement incident est transférée aux paires de Cooper qui se brisent en libérant chacune deux quasi-particules. Pour une énergie de $6 \mathrm{keV}$, quelques $10^{6}$ quasi-particules sont ainsi créées. Différents senseurs peuvent être utilisés pour mesurer ce nombre de quasi-particules : un thermomètre à transition supraconductrice ou une jonction tunnel supraconductrice par exemple.

Une jonction tunnel supraconductrice est constituée d'au moins deux couches supraconductrices séparées par une mince couche d'isolant (Fig. 2). Par un effet quantique, l'effet tunnel, les quasi-particules peuvent traverser cette barrière. Une couche supraconductrice supplémentaire, avec une bande d'énergie interdite plus faible, est souvent utilisée pour piéger les quasi-particules à proximité de la barrière, augmenter ainsi leur densité et favoriser leur passage. La jonction tunnel est polarisée. Le courant mesuré traversant la jonction est proportionnel au nombre de quasi-particules ayant traversé la barrière, et donc proportionnel à l'énergie déposée par un rayonnement ayant interagi dans la première couche supraconductrice.

La température de fonctionnement d'un tel détecteur doit être très inférieure à la température critique des matériaux supraconducteurs utilisés. Ceci est nécessaire afin d'éviter que l'excitation thermique ne brise les paires de Cooper et 
n'entre en compétition avec le phénomène recherché. La réinitialisation d'un tel détecteur peut être relativement rapide et des taux de comptage de l'ordre de 10000 évènements par seconde sont accessibles. Néanmoins les dimensions de l'absorbeur doivent être faibles pour permettre une diffusion des quasi-particules sans perte par recombinaison. Les détecteurs qui permettront d'obtenir une excellente résolution en énergie auront un rendement de détection limité.

Un thermomètre (ou senseur) à transition supraconductrice peut également être utilisé pour mesurer l'élévation de température consécutive à l'interaction d'un rayonnement. La température de fonctionnement doit être précisément celle où s'effectue la transition supraconductrice du senseur (souvent inférieure à une centaine de millikelvin). Dans ce cas, une très faible élévation de température se traduira par une forte variation de la résistance de la couche supraconductrice du senseur.

Les deux senseurs décrits précédemment ont une faible impédance. L'électronique la plus adaptée pour amplifier le signal est un SQUID, magnétomètre très sensible basé sur un phénomène quantique. Couplé à une bobine, le SQUID est utilisé comme amplificateur de courant avec un très faible niveau de bruit.

\subsection{Transfert d'énergie aux phonons}

Dans un diélectrique, l'énergie thermique est déterminée par les vibrations du réseau cristallin. Cette énergie est quantifiée. Les quanta, appelés phonons, portent une énergie de l'ordre de $10^{-5}$ à $10^{-4} \mathrm{eV}$ à basse température (inférieure à une centaine de millikelvin). Un rayonnement incident transfère donc son énergie à un très grand nombre de porteurs d'énergie. Pour une énergie incidente de $6 \mathrm{keV}$, environ $10^{8}$ phonons sont créés. Ces quanta peuvent être mesurés par un thermomètre à transition supraconductrice ou plus simplement par un thermomètre résistif réalisé à partir de germanium dopé par irradiation neutronique ou de silicium implanté. Cette technologie peut être utilisée pour la lecture d'un absorbeur de relativement grandes dimensions (ex. : saphir de $1 \mathrm{~kg}$ ). Chaque interaction se traduit par une variation de résistance électrique dont le temps de montée correspond à l'homogénéisation thermique du détecteur (quelques microsecondes à quelques centaines de microsecondes). Le signal étant proportionnel à l'énergie déposée et inversement proportionnel à la capacité calorifique, de très basses températures sont souhaitables (inférieures à une centaine de millikelvin). Par ailleurs la limite fondamentale de résolution en énergie d'un tel détecteur est donnée par les fluctuations thermodynamiques d'énergie entre le détecteur et le bain thermique : $\Delta \mathrm{E}_{\mathrm{FWHM}}=2,355\left(4 \mathrm{k}_{\mathrm{B}} \mathrm{T}^{2} \mathrm{C}\right)^{1 / 2}$, 


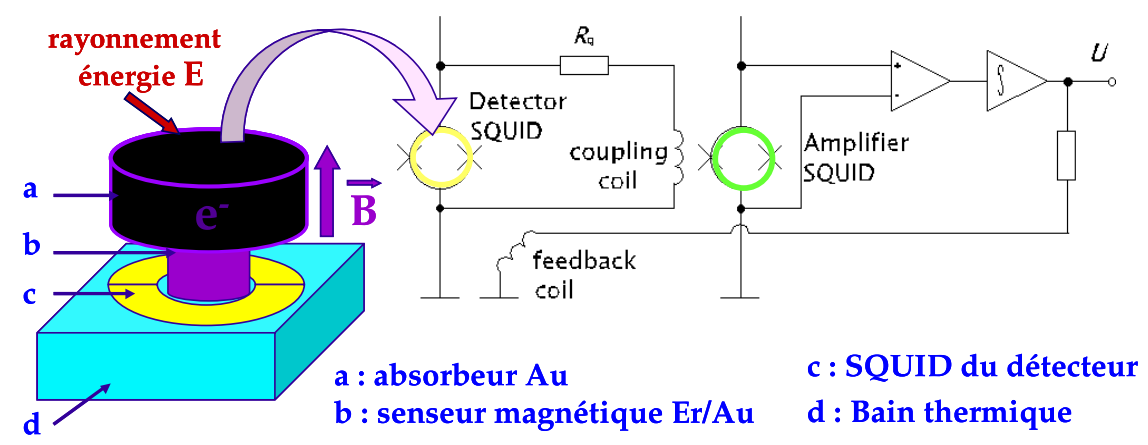

Figure 3 - Microcalorimètre magnétique.

Magnetic microcalorimeter.

où $\mathrm{k}_{\mathrm{B}}$ est la constante de Boltzmann, $\mathrm{T}$ la température et $\mathrm{C}$ la capacité calorifique $\mathrm{du}$ détecteur. Les thermomètres en germanium ou en silicium dopés ont une impédance élevée ( 1 à $100 \mathrm{M} \Omega$ ) et nécessitent, comme premier étage d'amplification, un transistor à effet de champ (FET), refroidi ou non.

\subsection{Transfert d'énergie aux électrons de conduction}

Dans un métal à basse température, un rayonnement incident transfère son énergie aux électrons de conduction, il en résulte une élévation de température. L'énergie moyenne cédée à chaque électron est très faible. En conséquence, quelle que soit l'énergie déposée, un très grand nombre de porteurs d'énergie est créé, de plusieurs ordres de grandeur supérieur au nombre de phonons créés dans le cas d'un isolant. La lecture de cette élévation de température peut se faire par un thermomètre magnétique. Celui-ci est constitué d'une matrice métallique dans laquelle sont distribués des ions paramagnétiques (erbium dans or, par exemple). Soumis à un champ magnétique, le thermomètre traduit une élévation de température par une variation de moment magnétique. Le thermomètre peut être couplé, ou directement placé, dans la boucle d'un SQUID, magnétomètre très sensible. Le signal mesuré est donc une variation de flux magnétique, proportionnelle à l'énergie déposée par le rayonnement incident dans le métal. Une façon élégante d'amplifier le signal est d'utiliser un deuxième SQUID qui constitue un étage d'amplification à très faible bruit (Fig. 3). La technologie des microcalorimètres magnétiques est récente (quelques années) mais a déjà démontré les meilleures performances en terme de résolution en énergie. 
Tableau I

Comparaison des différents principes physiques des détecteurs conventionnels et cryogéniques. Comparison of the different physics principles of conventional and cryogenic detectors.

\begin{tabular}{lcc}
\hline Détecteur & $\begin{array}{c}\text { Quanta auxquels l'énergie } \\
\text { est transférée }\end{array}$ & Énergie portée par un quantum \\
\hline Scintillateur & photons UV, visible & 0,1 à $1 \mathrm{keV}$ \\
Compteur proportionnel & ionisation & $10 \mathrm{à} 30 \mathrm{eV}$ \\
Semi-conducteur & paires electron-trou & $3-4 \mathrm{eV}$ \\
\hline$\grave{A}$ très basse température $<100 \mathrm{mK}$ & & \\
\hline Jonction tunnel & quasi-particules & $10^{-3} \mathrm{eV}$ \\
Calorimètre diélectrique & phonons & $10^{-5}$ à $10^{-4} \mathrm{eV}$ \\
Calorimètre magnétique & électrons de conduction & $<<10^{-5} \mathrm{eV}$ \\
\hline
\end{tabular}

\subsection{Comparaison avec les détecteurs conventionnels}

Le tableau I récapitule les différents principes physiques exploités par les détecteurs conventionnels et les détecteurs cryogéniques. Ces derniers mettent en jeu des quanta (quasi-particules, phonons, électrons) portant chacun en moyenne une énergie très faible. L'énergie à mesurer est donc distribuée sur un très grand nombre de porteurs, d'où une très faible fluctuation statistique, permettant un seuil de détection très bas et une excellente résolution en énergie, meilleure de plus d'un ordre de grandeur par rapport aux détecteurs classiques. Les différentes générations de détecteurs cryogéniques ont introduit une rupture technologique pour la mesure des rayonnements ionisants équivalente à celle apportée par les détecteurs semi-conducteurs dans les années 1970. Si le principe physique permet de comprendre pourquoi il est possible de repousser de plusieurs ordres de grandeur les limites des détecteurs conventionnels, qu'en est-il des performances réelles obtenues avec ces nouvelles technologies?

\section{3. État de l'art en spectrométrie $X$}

Les résolutions en énergie publiées par différentes équipes sont remarquables et approchent les limites théoriques annoncées par le principe physique (Tab. II). La meilleure résolution en énergie, associée à un rendement de détection proche de $100 \%$ pour des photons de $6 \mathrm{keV}$ est égale à $3,4 \mathrm{eV}$. Ce très beau résultat a été obtenu par l'université de Heidelberg avec un microcalorimètre magnétique lu par une électronique à deux SQUIDs (Fleischmann et al., 2004).

L'état de l'art en spectrométrie $\mathrm{X}$ ne se limite pas à la réalisation de détecteurs individuels. Depuis quelques années déjà des mosaïques de détecteurs basées sur les technologies les plus anciennes (excitation de quasi-particules ou de phonons) 
TABLEAU II

Performances publiées en spectrométrie $X$.

Published performances in X-ray spectrometry.

\begin{tabular}{|c|c|c|c|}
\hline Institut & Detecteur & $\begin{array}{l}\text { Résolution en énergie } \\
\text { à } 6 \mathrm{keV} \text { pour un pixel }\end{array}$ & $\begin{array}{l}\text { Résolution en énergie } \\
\text { à } 6 \mathrm{keV} \text { (mosaïque) }\end{array}$ \\
\hline $\begin{array}{l}\text { Technical Univ. of Munich, } \\
\text { Germany } \\
\text { (Huber et al., 2004) }\end{array}$ & $\begin{array}{l}\text { Absorbeur : Pb } \\
\text { senseur : Al-AlO }-A l \text { STJ }\end{array}$ & $12 \mathrm{eV}$ & \\
\hline $\begin{array}{l}\text { ISAS, Mitsubishi, } \\
\text { Panasonic, Seiko Instr. (3): } \\
\text { NEXT (Fujimoto et al., } \\
\text { 2004) }\end{array}$ & $\begin{array}{l}\text { Absorbeur : Sn ou Bi } \\
\text { Senseur : Ti/Au TES }\end{array}$ & $6,3 \mathrm{eV}$ & prochain objectif 1000 pixels \\
\hline $\begin{array}{l}\text { Univ. of Wisconsin; NASA, } \\
\text { Greenbelt, USA: XRS } \\
\text { (Brekosky et al., 2004) }\end{array}$ & $\begin{array}{l}\text { Absorbeur : HgTe } \\
\text { senseur : doped Si }\end{array}$ & $6 \mathrm{eV}$ & $\begin{array}{l}\mathbf{6} \text { eV sur } 4 \text { réseaux de } 36 \text { pixels } \\
\text { prochain objectif } 1000 \text { pixels }\end{array}$ \\
\hline $\begin{array}{l}\text { NASA, Goddard Space } \\
\text { Flight Center, USA: } \\
\text { Con-X (Lindeman et al., } \\
\text { 2004) }\end{array}$ & $\begin{array}{l}\text { Absorbeur : Bi/Cu } \\
\text { senseur : Au/Mo TES }\end{array}$ & & $\begin{array}{l}7 \mathrm{eV} \text { sur } 25 \text { pixels, } \\
\text { prochain objectif } 1000 \text { pixels }\end{array}$ \\
\hline $\begin{array}{l}\text { NIST, USA } \\
\text { PTB, Germany } \\
\text { (Hilton et al., 2004) }\end{array}$ & $\begin{array}{l}\text { Absorbeur/senseur : } \\
\text { Mo/Cu TES } \\
\text { Absorbeur : Bi } \\
\text { Senseur : Al/Ag TES }\end{array}$ & $\begin{array}{c}4,5 \mathrm{eV} \\
2,0 \mathrm{eV} \text { à } 1,5 \mathrm{keV}\end{array}$ & $22 \mathrm{eV}$ sur 64 pixels \\
\hline $\begin{array}{l}\text { SRON, MESA, } \\
\text { Netherlands, Finland: } \\
\text { XEUS (Bruijin et al., } \\
\text { 2004) }\end{array}$ & $\begin{array}{l}\text { Absorbeur : } \mathrm{Cu}, \\
\text { senseur : Au/Ti TES }\end{array}$ & $4,5 \mathrm{eV}$ & $\begin{array}{l}6 \mathrm{eV} \text { sur } 25 \text { pixels } \\
\text { prochain objectif } 1000 \text { pixels }\end{array}$ \\
\hline $\begin{array}{l}\text { Univ. of Heidelberg, } \\
\text { Germany } \\
\text { (Fleischmann et al., 2004) }\end{array}$ & $\begin{array}{l}\text { Absorbeur : Au } \\
\text { Senseur : Au/Er } \\
\text { magnétique }\end{array}$ & $\begin{array}{l}3,4 \mathrm{eV} \text { rendement de } \\
\text { détection }>99 \%\end{array}$ & à l'étude \\
\hline
\end{tabular}

sont conçues et testées. Les résultats publiés pour des matrices de quelques dizaines de pixels démontrent le potentiel des détecteurs cryogéniques (Tab. II). Les développements actuels portent sur la construction de mosaïques de plus de 1000 pixels. L'objectif à long terme est de réaliser des spectromètres imageurs de plus de 1 million de pixels.

\section{Qu'en est-il de la spectrométrie gamma?}

Les détecteurs cryogéniques ont été relativement peu explorés pour la spectrométrie gamma. Néanmoins, les quelques résolutions en énergie publiées pour des photons de $60 \mathrm{keV}$ sont déjà intéressantes, de l'ordre de 60 à 70 eV (Bleile et al., 2002 ; Silver et al., 2002). Les détecteurs qui donnent la possibilité d'utiliser des absorbeurs de densité élevée et à fort numéro atomique sont potentiellement les plus intéressants pour la spectrométrie gamma. D’une part, le rendement de détection sera important pour des dimensions réduites du détecteur. D'autre part, l'effet photoélectrique étant proportionnel à $\mathrm{Z}^{4,5}$, le rapport de la surface du pic 
d'absorption totale sur le fond Compton sera excellent. C'est le cas, par exemple, des calorimètres magnétiques qui mettent en œuvre une cible en or de forte masse volumique $\left(19 \mathrm{~g} / \mathrm{cm}^{3}\right)$ et de numéro atomique très élevé $(\mathrm{Z}=79)$, offrant un avantage remarquable par rapport aux détecteurs en germanium classiques (masse volumique $5 \mathrm{~g} / \mathrm{cm}^{3}$ et $\mathrm{Z}=32$ ). La diminution du fond Compton et l'amélioration de la résolution en énergie autorisent un abaissement des limites de détection. Les calorimètres magnétiques ont ainsi la potentialité de donner accès à des informations qui étaient jusqu'à aujourd'hui inaccessibles.

\section{Applications d'aujourd'hui et de demain}

\subsection{Métrologie des rayonnements ionisants}

La gestion des déchets nucléaires, le contrôle de l'environnement et les applications médicales de la radioactivité notamment nécessitent de disposer de références ou étalons de radioactivité dont l'activité est déterminée avec l'incertitude la plus faible possible. La mesure précise de radio-nucléides émetteurs de faible énergie ou à vie longue pose un problème en raison de la possibilité d'auto-absorption du rayonnement dans la source et de l'effondrement du rendement de détection de la plupart des détecteurs pour les électrons et les photons $\mathrm{X}$ de basse énergie. Le Bureau national de métrologie/Laboratoire national Henri Becquerel (BNM-LNHB) a démontré que les détecteurs cryogéniques offrent une alternative incomparable pour la mesure d'activité, donnant accès à un rendement de détection proche de $100 \%$ pour des électrons et des $\mathrm{X}$ à partir du seuil de quelques dizaines d'électron-volts et ceci jusqu'à $10 \mathrm{keV}$ (Loidl et al., 2004). Le détecteur utilisé pour cette étude est un microcalorimètre magnétique dont l'absorbeur est une feuille d'or enfermant en géométrie $4 \pi$ la source à mesurer. Le spectre d'absorption totale obtenu pour une source de ${ }^{55} \mathrm{Fe}$ est conforme au spectre théorique attendu. Au-delà de la mesure d'activité, les détecteurs cryogéniques ouvrent de nouvelles perspectives dans la métrologie des rayonnements ionisants en donnant accès à la mesure directe de données atomiques ou nucléaires, par exemple les probabilités de capture électronique.

\subsection{Applications dans la recherche}

- La plupart des objets observables en astronomie émettent naturellement un rayonnement $X$ ou peuvent être étudiés par leur absorption de rayonnement $X$. Un spectromètre imageur d'excellente résolution en énergie et de large bande spectrale introduirait une révolution dans l'enregistrement des données et la compréhension des phénomènes étudiés. Ceci explique l'enjeu considérable lié à la construction de mosaïques de détecteurs cryogéniques et l'implication de nombreuses équipes telles que NIST, NASA, Stanford University, etc. pour 
préparer les futures missions spatiales embarquées XRS sur Astro-E2 (Brekosky et al., 2004), XEUS (Bruijn et al., 2004) et NEXT (Fujimoto et al., 2004) (1024 pixels, FWHM $=3 \mathrm{eV}$ à $6 \mathrm{keV}$, 2010). L'étude du système solaire et de la cosmologie mobilisent également des forces pour la construction de caméras pour des rayonnements sub-millimétriques (SHARC-II, SAMBA, SCUBA) et microondes pour l'étude du fond cosmologique (POLARBEAR, GBT), de télescopes infrarouge (SAFIR) ou de caméras infrarouge (SPEED). Des caméras pour la mesure des photons optiques et UV sont également développées (Martin et al., 2004 ; Burney et al., 2004), en particulier par l'ESA et le NIST.

- Pour la détermination de données atomiques telles que les sections efficaces d'interaction absolues, des mosaïques constituées de 32 microcalorimètres avec une résolution en énergie égale à $11 \mathrm{eV}$ pour des photons de $6 \mathrm{keV}$ sont mises en œuvre (NASA, LLNL) pour l'étude de l'émission $\mathrm{X}$ de plasmas thermiques.

- Les recherches sur la détermination de la masse du neutrino mettent en œuvre différents types de détecteurs cryogéniques. Par exemple l'INFN utilise des matrices de 10 microcalorimètres en $\mathrm{AgReO}_{4}$ pour l'étude du spectre bêta de ${ }^{187} \mathrm{Re}$ afin de repousser les limites sur la détermination de la masse de l'antineutrino électronique. Par ailleurs une large collaboration impliquant l'Italie et les USA étudie la double désintégration bêta de ${ }^{130} \mathrm{Te}$ en mettant en œuvre une mosaïque de 60 détecteurs de $\mathrm{TeO}_{2}$ avec une masse totale d'environ $40 \mathrm{~kg}$. L'objectif final est de construire un réseau de 1000 détecteurs de $760 \mathrm{~g}$ chacun.

- Les détecteurs cryogéniques ont permis de repousser les limites reconnues jusqu'à ce jour pour la sensibilité de détection de particules susceptibles de constituer ce qu'on appelle la matière noire. Différentes technologies sont mises en œuvre. Dans tous les cas, il s'agit d'absorbeurs massifs (quelques centaines de grammes à $1 \mathrm{~kg}$ ). La détection de la chaleur est couplée à une détection simultanée de l'ionisation ou de la scintillation dans l'absorbeur afin de pouvoir discriminer efficacement un recul de noyau (signature probable de l'interaction d'une particule recherchée), du bruit de fond gamma. Ces détecteurs permettent également une excellente discrimination entre l'interaction d'une particule alpha et celle d'un photon de même énergie. Utilisé dans un environnement de très faible bruit de fond radioactif, un tel détecteur a permis au CNRS/IAS de découvrir la radioactivité naturelle de ${ }^{209} \mathrm{Bi}$ que l'on pensait stable jusqu'à ce jour en raison de sa très longue période (De Marcillac et al., 2003).

- Les détecteurs cryogéniques ont également démontré d'excellentes performances pour la spectrométrie de masse d'ions lourds avec des résolutions en énergie relative de l'ordre de $10^{-3}$ à $1 \mathrm{MeV}$. Un des objectifs est de déterminer avec une très grande sensibilité le rapport isotopique ${ }^{236} \mathrm{U} /{ }^{238} \mathrm{U}$ d'échantillons naturels. Un rapport de $7 \times 10^{-12}$ a été mesuré (Kraft et al., 2004). 
- Un spectromètre imageur pour la mesure des neutrons est à l'étude au Japon (Nakamura et al., 2004). Le détecteur est réalisé avec un absorbeur en $\mathrm{Li}_{2} \mathrm{~B}_{4} \mathrm{O}_{7}$ couplé à des jonctions tunnel. Le LLNL (USA) développe également un spectromètre neutron avec un absorbeur LiB couplé à un thermomètre à transition supraconductrice.

\subsection{Applications dans l'industrie}

- Des détecteurs cryogéniques sont actuellement commercialisés pour l'analyse de matériaux par fluorescence $X$ (Hollerith et al., 2004). Les résolutions en énergie sont de l'ordre de $20 \mathrm{eV}$ à $6 \mathrm{keV}$ et $10 \mathrm{eV}$ à $1,5 \mathrm{keV}$ apportant une sensibilité remarquable, appréciée entre autres, par les constructeurs de semi-conducteurs pour l'analyse d'impuretés.

- À l'issue d'une collaboration fructueuse (Neuchâtel, NIST Boulder, et GenSpec SA) le premier spectromètre de masse par temps de vol utilisant un détecteur cryogénique est commercialisé ${ }^{2}$ et a été testé avec succès pour des bio-molécules (Rutzinger et al., 2004). Les applications sont par exemple l'identification de protéines, la détection de virus, l'étude de séquences ADN, ... La spectrométrie d'absorption $\mathrm{X}$ est également envisagée pour le suivi de réactions biochimiques et l'analyse d'éléments métalliques.

- Les applications de demain dans l'industrie seront celles qui exigent de franchir les limites de détection des détecteurs conventionnels. Elles concerneront notamment les domaines de la sécurité (la non-prolifération de matières nucléaires, la détection d'explosifs, la recherche d'agents chimiques), l'environnement (suivi de la qualité de l'air, contrôle des eaux), et le cycle du combustible (analyse isotopique de plutonium et d'uranium, suivi des déchets nucléaires). La médecine bénéficiera également des possibilités des détecteurs cryogéniques en particulier pour la microscopie associée à la spectrométrie de photons X (Friedrich, 2004) et l'imagerie par tomographie (positron emission tomography) (Dawson et al., 2004).

\section{Conclusions}

Les détecteurs cryogéniques, dont le principe de détection est fondé sur la mesure d'une élévation de température, présentent deux avantages. L'énergie d'un rayonnement incident, se retrouvant essentiellement sous forme de chaleur, est convertie très efficacement en énergie détectable. De plus, cette énergie est

2 Comet Analytics, 1 rue Pourtalés, 2000 Neuchâtel, Suisse. 
distribuée sur un très grand nombre de porteurs d'énergie entraînant de très faibles fluctuations statistiques, donc une très grande précision sur la détermination de l'énergie, donnant accès à un très faible seuil de détection ainsi qu'à une excellente résolution en énergie. Ceci explique pourquoi les performances des trois grandes classes de détecteurs cryogéniques ont déjà repoussé de un à deux ordres de grandeur les limites des détecteurs conventionnels, en ce qui concerne le seuil de détection et la résolution en énergie. Le développement des détecteurs cryogéniques est aujourd'hui en expansion pour des application très diverses dans les domaines de la recherche. Le coût décroissant de ces détecteurs ainsi que la commercialisation très récente de nouveaux réfrigérateurs ouvrent de nouvelles perspectives d'applications en particulier dans l'industrie. En effet, il existe aujourd'hui des réfrigérateurs de faible encombrement (similaire à un Dewar d'azote liquide pour refroidir un détecteur à semi-conducteur), et fonctionnant sans liquide cryogénique (le fonctionnement des tubes pulsés ne requiert qu'une alimentation secteur). La température de base $(50$ à $100 \mathrm{mK})$ est obtenue par désaimantation adiabatique. La simple commutation d'un interrupteur permet de refroidir le détecteur de la température ambiante à la température de base en quelques heures. Contrairement aux détecteurs à semi-conducteurs, les détecteurs cryogéniques ne nécessitent pas d'être refroidis en permanence. Ils peuvent être réchauffés lorsqu'ils ne sont pas utilisés et refroidis uniquement aux moments des mesures, ce qui représente un avantage pour des applications industrielles. Aujourd'hui, seuls des microcalorimètres pour la spectrométrie des photons X sont commercialisés intégrés dans un tel réfrigérateur ${ }^{3}$.

\section{RÉFÉRENCES}

Bleile A., Egehof P., Kraft S., Mc Cammon D., Meier H. J., Shrivastava A., Stahle C.K., Weber W. (2002) Calorimetric low temperature Detectors for high resolution X-ray spectroscopy on stored highly stripped heavy ions, Proceedings of the 9th International Workshop on Low Temperature Detectors, Madison, Wisconsin, 2001 (F.S. Porter et al., Eds.), AIP Conference Proceedings, Melville, New York, pp. 409-412.

Brekosky R. et al. (2004) Fabrication processes responsible for fundamentally improving silicon X-ray microcalorimeter arrays, Proceedings of the 10th International Workshop on Low Temperature Detectors, Genoa, Italy, July 2003 (F. Gatti, Ed.), Nucl. Instr. Meth. A 520, 439-442.

Bruijn M.P. et al. (2004) Development of an array of transition edge sensors for applications in X-ray astronomy, Proceedings of the 10th International Workshop on Low Temperature Detectors, Genoa, Italy, July 2003 (F. Gatti, Ed.), Nucl. Instr. Meth. A 520, 443-445.

Burney J. et al. (2004) Development and characterization of a TES optical imaging array for astrophysics applications, Proceedings of the 10th International Workshop on Low Temperature Detectors, Genoa, Italy, July 2003 (F. Gatti, Ed.), Nucl. Instr. Meth. A 520, 533-536.

\footnotetext{
3 Janis Research Company, Inc., 2 Jewel Drive, P.O. Box 696, Wilmington, MA 01887-0696 USA, www.janis.com/p adr.html et VeriCold Technologies GmbH, Bahnhofstraße 21, D-85737 Ismaning, Germany, www.vericold.com.
} 


\section{DÉVELOPPEMENTS RÉCENTS SUR LES DÉTECTEURS CRYOGÉNIQUES}

Dawson R.B., Maillard J., Maurel G., Silva J., Waysand G. (2004) Superheated superconducting grains (SSG) in high density dielectric matrix (HDDM): feasibility of a positron emission tomography, Proceedings of the 10th International Workshop on Low Temperature Detectors, Genoa, Italy, July 2003 (F. Gatti, Ed.), Nucl. Instr. Meth. A 520, 628-630.

De Marcillac P. et al. (2003) Experimental detection of alpha particles from the radioactive decay of natural bismuth, Nature 422, 876-878.

Fleischmann A., Link M., Daniyarov T., Rotzinger H., Enss C., Seidel G.M. (2004) Metallic magnetic calorimeters: detectors for high resolution X-ray spectroscopy, Proceedings of the 10th International Workshop on Low Temperature Detectors, Genoa, Italy, July 2003 (F. Gatti, Ed.), Nucl. Instr. Meth. A 520, 27-31.

Friedrich S. (2004) Biological applications of cryogenic detectors, Proceedings of the 10th International Workshop on Low Temperature Detectors, Genoa, Italy, July 2003 (F. Gatti, Ed.), Nucl. Instr. Meth. A 520, 621-624.

Fujimoto R. et al. (2004) TES microcalorimeter development for future japanese X-ray astronomy missions, Proceedings of the 10th International Workshop on Low Temperature Detectors, Genoa, Italy, July 2003 (F. Gatti, Ed.), Nucl. Instr. Meth. A 520, 431-434.

Hilton G.C. et al. (2004) Fabrication of the SCUBA-2 detector arrays, Proceedings of the 10th International Workshop on Low Temperature Detectors, Genoa, Italy, July 2003 (F. Gatti, Ed.), Nucl. Instr. Meth. A 520, 483-486.

Hollerith C. et al. (2004) Energy dispersive X-ray spectroscopy with microcalorimeters, Proceedings of the 10th International Workshop on Low Temperature Detectors, Genoa, Italy, July 2003 (F. Gatti, Ed.), Nucl. Instr. Meth. A 520, 606-609.

Huber M. et al. (2004) Characterization of Al-STJ based X-ray detector with monochromatized synchrotron radiation, Proceedings of the 10th International Workshop on Low Temperature Detectors, Genoa, Italy, July 2003 (F. Gatti, Ed.), Nucl. Instr. Meth. A 520, 234-236.

Kraft S. et al. (2004) First application of calorimetric low temperature detectors in accelerator mass spectrometry, Proceedings of the 10th International Workshop on Low Temperature Detectors, Genoa, Italy, July 2003 (F. Gatti, Ed.), Nucl. Instr. Meth. A 520, 63-66.

Lindeman M.A. et al. (2004) Performance of compact TES-arrays with integrated high fill fraction Xray absorbers, Proceedings of the 10th International Workshop on Low Temperature Detectors, Genoa, Italy, July 2003 (F. Gatti, Ed.), Nucl. Instr. Meth. A 520, 411-413.

Loidl M., Leblanc E., Branger T., Rotzinger H., Daniyarov T., Linck M., Fleischmann A., Enss C. (2004) Feasibility study of absolute activity measurement with metallic magnetic microcalorimeters, Proceedings of the 10th International Workshop on Low Temperature Detectors, Genoa, Italy, July 2003 (F. Gatti, Ed.), Nucl. Instr. Meth. A 520, 73-75.

Martin D., Verhoeve P., Dordrecht A., Verveer J., Hijmering R. (2004) A 12x10 pixels superconducting tunnel junction array based spectrophotometer for optical astronomy, Proceedings of the 10th International Workshop on Low Temperature Detectors, Genoa, Italy, July 2003 (F. Gatti, Ed.), Nucl. Instr. Meth. A 520, 512-515.

Nakamura T., Katagiri M., Ukibe M., Ikeuchi T., Ohkubo M. (2004) Neutron detection by superconducting tunnel junctions with $\mathrm{Li}_{2} \mathrm{~B}_{4} \mathrm{O}_{7}$ single crystal absorber, Proceedings of the 10th International Workshop on Low Temperature Detectors, Genoa, Italy, July 2003 (F. Gatti, Ed.), Nucl. Instr. Meth. A 520, 67-69.

Rutzinger S., Christ P., Pröbst F., Seidel W., Uchaikin S., Stark M. (2004) Development of a superconducting-phase-transition thermometer (SPT) for the application in a time-of-flight mass spectrometer (TOF-MS) for heavy-mass molecules, Proceedings of the 10th International Workshop on Low Temperature Detectors, Genoa, Italy, July 2003 (F. Gatti, Ed.), Nucl. Instr. Meth. A 520, 625-627.

Silver E. et al. (2002) X-ray and gamma-ray astronomy with NTD germanium-based microcalorimeters, Proceedings of the 9th International Workshop on Low Temperature Detectors, Madison, Wisconsin, 2001 (F.S. Porter et al., Eds.) AIP Conference Proceedings, Melville, New York, pp. 555-558 